\title{
Unambiguous SAR Signal Reconstruction From Nonuniform Displaced Phase Center Sampling
}

\author{
Gerhard Krieger, Member, IEEE, Nicolas Gebert, and Alberto Moreira, Senior Member, IEEE
}

\begin{abstract}
The displaced phase center (DPC) technique will enable a wide-swath synthetic aperture radar (SAR) with high azimuth resolution. In a classic DPC system, the pulse repetition frequency (PRF) has to be chosen such that the SAR carrier moves just one half of its antenna length between subsequent radar pulses. Any deviation from this PRF will result in a nonuniform sampling of the synthetic aperture. This letter derives an innovative reconstruction algorithm and shows that an unambiguous reconstruction of a SAR signal is possible for nonuniform sampling of the synthetic aperture. This algorithm will also have great potential for multistatic satellite constellations as well as the dual receive antenna mode in Radarsat 2 and TerraSAR-X.
\end{abstract}

Index Terms-Azimuth ambiguities, digital beamforming, displaced phase center antenna (DPCA), high-resolution wide-swath SAR, interferometry, synthetic aperture radar (SAR).

\section{INTRODUCTION}

W IDE-SWATH imaging and high azimuth resolution pose contradicting requirements on synthetic aperture radar (SAR) system design. To overcome this fundamental limitation, several innovative techniques have been suggested that use multiple receiver apertures to acquire additional samples along the synthetic aperture. The apertures may be either on a single platform like in the classical displaced phase center (DPC) technique [1]-[6] or on different platforms [7]-[9] leading to a multistatic SAR where the size of each individual receiver is reduced. Fig. 1 shows one example for each scenario, where a single transmitter illuminates a wide swath and $n$ subapertures record simultaneously the scattered signal from the illuminated footprint. Under ideal conditions, this will allow for a reduction of the pulse repetition frequency (PRF) by a factor of $n$ without rising azimuth ambiguities. This reduction of the azimuth sampling rate becomes possible by a coherent combination of the individual receiver signals where the ambiguous parts of the Doppler spectra cancel each other. Note that such an ambiguity suppression can also be regarded as digital beamforming on receive where nulls in the joint antenna pattern are steered to the ambiguous zones. For optimum performance, the along-track displacement of the subapertures $i=\{2, \ldots, n\}$ relative to the first receiver $(i=1)$ should be chosen as

$$
x_{i}-x_{1} \approx \frac{2 v}{\operatorname{PRF}}\left(\frac{i-1}{n}+k_{i}\right), \quad k_{i} \in Z
$$

which will result in a uniform sampling of the received SAR signal. In this equation, PRF is the pulse repetition frequency of the transmitter and $v$ is the velocity of the SAR carrier. Note that for a multistatic constellation the $k_{i}$ may be different for

Manuscript received March 24, 2004; revised May 13, 2004.

The authors are with the Microwaves and Radar Institute, German Aerospace Centre (DLR), D-82234 Wessling, Germany (e-mail: Gerhard.Krieger@ dlr.de). Digital Object Identifier 10.1109/LGRS.2004.832700
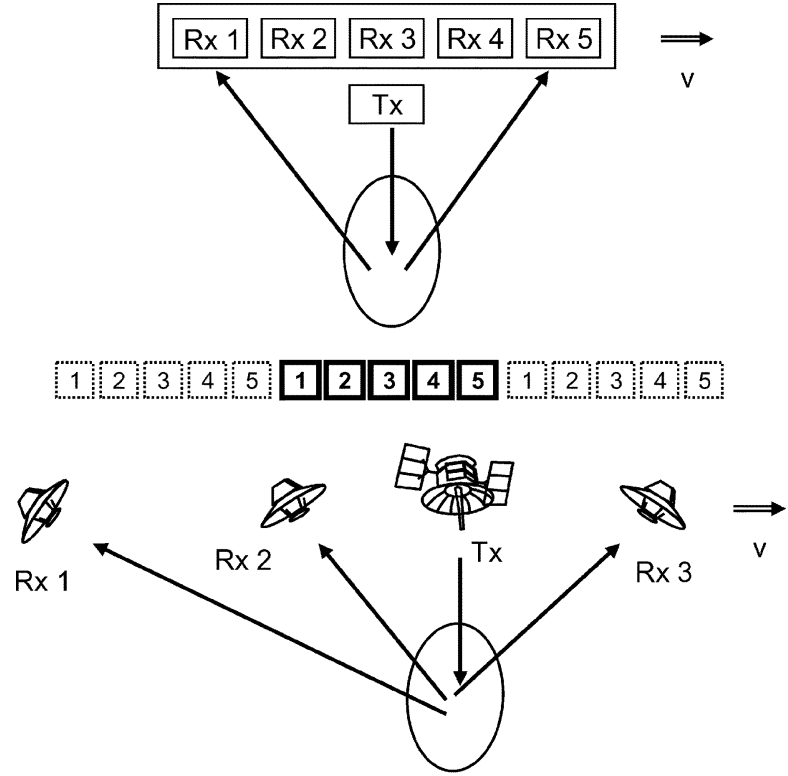

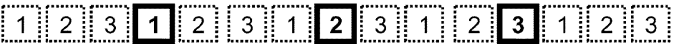

Fig. 1. Multiple aperture sampling for a single-platform system (top) and a distributed satellite array (bottom). The effective (bistatic) phase centers are shown as squares. Solid squares correspond to samples of the synthetic aperture for the illustrated transmitter (Tx) and receiver ( $\mathrm{Rx})$ positions. The dotted squares are for previous and subsequent positions assuming an appropriately chosen PRF.

each receiver, which enables a great flexibility in choosing the along-track distance between the satellites. In a single-platform system, all $k_{i}$ will be zero. Since the subaperture distance and the platform velocity are fixed in this case, a specific PRF will be required

$$
\mathrm{PRF}=\frac{2 \cdot v}{n \cdot \Delta x}
$$

where we assume an antenna with $n$ subaperture elements separated by $\Delta x=x_{i+1}-x_{i}$. The PRF in a single-platform DPC system has thus to be chosen such that the SAR platform moves just one half of the total antenna length between subsequent radar pulses. However, such a rigid selection of the PRF may be in conflict with the timing diagram for some incident angles. It will, furthermore, exclude the opportunity to use an increased PRF for improved azimuth ambiguity suppression.

\section{General ApProACH}

In the following, an innovative reconstruction algorithm will be derived, which allows for an unambiguous recovery of the Doppler spectrum even for a nonuniform sampling of the SAR signal. The only requirement is that the samples do 

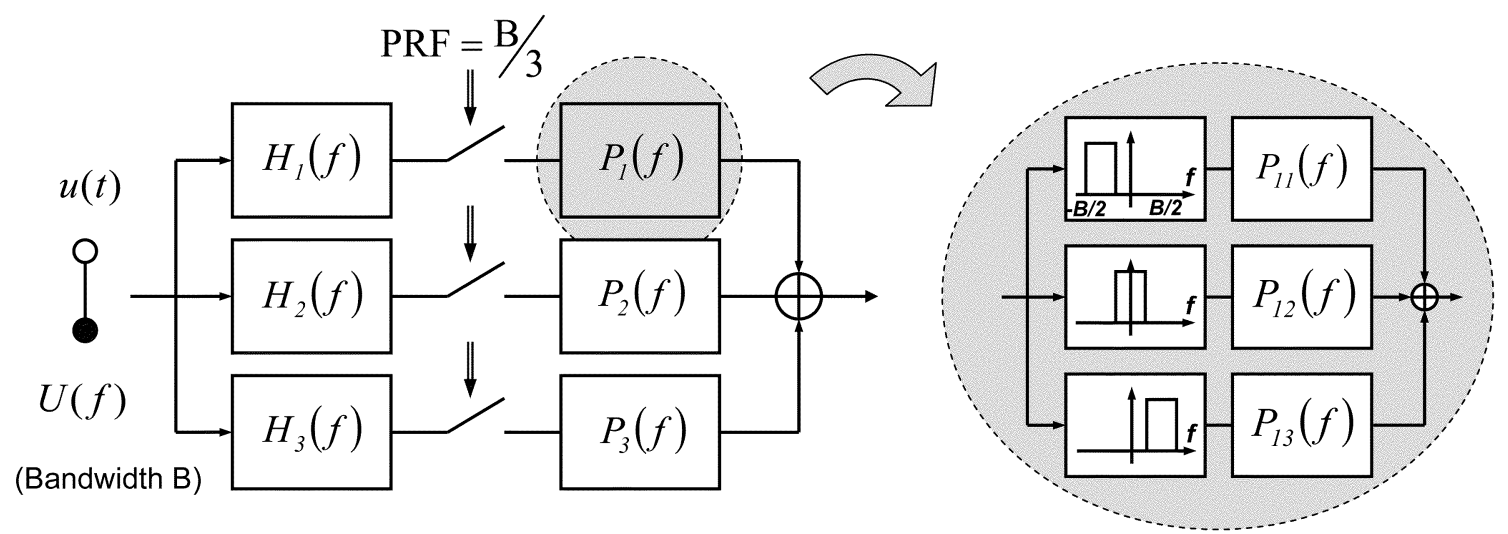

Fig. 2. (Left) Reconstruction for multichannel subsampling in case of three channels. (Right) Each reconstruction filter $P_{i}$ consists of $n$ bandpass filters $P_{i j}$.

not coincide. Such an algorithm has a great potential for any multiaperture system, be it multistatic with sparsely distributed receiver satellites or be it a classic DPC antenna (DPCA) system like Radarsat 2 [5] or TerraSAR-X [6]. The data acquisition in a multiaperture SAR can be considered as a linear system with multiple receiver channels, each described by a linear filter $h_{i}(t)$ with transfer function $H_{i}(f)$ (cf. Fig. 2). For such a system, there exist many powerful theorems in linear systems theory. Of special importance for the present context is a generalization of the sampling theorem according to which a bandlimited signal $u(t)$ is uniquely determined in terms of the samples $h_{i}(n T)$ of the responses $h_{i}(t)$ of $n$ linear systems with input $u(t)$ sampled at $1 / n$ of the Nyquist frequency [10]. To be valid, the transfer functions of the linear filters may be selected in a quite general sense, but not arbitrarily (for details see [10]). A block diagram for the reconstruction from the subsampled signals is shown in Fig. 2. The reconstruction consists essentially of $n$ linear filters $P_{i}(f)$ that are individually applied to the subsampled signals of the receiver channels and then superimposed. Each of the reconstruction filters $P_{i}(f)$ can again be regarded as a composition of $n$ bandpass filters $P_{i j}(f)$, where $1 \leq j \leq n$. As shown in [10], the reconstruction filters can be derived from a matrix $\mathbf{H}(\mathbf{f})$ consisting of the $n$ transfer functions $H_{i}(f)$, which have to be shifted by integer multiples of the PRF in the frequency domain $\mathbf{H}(\mathbf{f})$

$$
=\left[\begin{array}{ccc}
H_{1}(f) & \cdots & H_{n}(f) \\
H_{1}(f+\mathrm{PRF}) & \cdots & H_{n}(f+\mathrm{PRF}) \\
\vdots & \ddots & \vdots \\
H_{1}(f+(n-1) \cdot \mathrm{PRF}) & \cdots & H_{n}(f+(n-1) \cdot \mathrm{PRF})
\end{array}\right] .
$$

The reconstruction filters $P_{i j}(f)$ are then derived from an inversion of the matrix $\mathbf{H}(\mathbf{f})$ as

$$
\begin{aligned}
& \mathbf{H}^{\mathbf{- 1}}(\mathbf{f}) \\
& =\left[\begin{array}{cccc}
P_{11}(f) & P_{12}(f+\mathrm{PRF}) & \cdots & P_{1 n}(f+(n-1) \mathrm{PRF}) \\
P_{21}(f) & P_{22}(f+\mathrm{PRF}) & \cdots & P_{2 n}(f+(n-1) \mathrm{PRF}) \\
\vdots & \vdots & \ddots & \vdots \\
P_{n 1}(f) & P_{n 2}(f+\mathrm{PRF}) & \cdots & P_{n n}(f+(n-1) \mathrm{PRF})
\end{array}\right] .
\end{aligned}
$$

\section{QUADRATIC Phase APPROXIMATION}

In principle, it is possible to use the multichannel SAR signal model for a complete reconstruction of the scene reflectivity.
However, in order to concentrate on the essential steps, we will only consider the azimuth modulation in the following derivation. The azimuth signal of a point target at azimuth time $t=0$ and slant range $r_{0}$ for each individual receiver channel $i$ may be described as

$$
\begin{aligned}
& h_{i}\left(t ; \Delta x_{i}\right)=A_{T x}(v t) \cdot A_{R x, i}(v t) \\
& \quad \cdot \exp \left[-j \frac{2 \pi}{\lambda}\left(\sqrt{r_{0}^{2}+(v t)^{2}}+\sqrt{r_{0}^{2}+\left(v t-\Delta x_{i}\right)^{2}}\right)\right]
\end{aligned}
$$

where $A_{T x}$ and $A_{R x, i}$ define the envelope of the azimuth signal arising from the projection of the transmit and receive antenna patterns on the ground. The phase of the azimuth signal is proportional to the sum of the transmit and receive paths that are here approximated by the two square roots assuming a straight flight path with velocity $v$. A further simplification arises if we expand (5) in a Taylor series up to the second order that will lead to the quadratic approximation

$$
\begin{aligned}
& h_{i}\left(t ; \Delta x_{i}\right) \cong A_{T x}(v t) \cdot A_{R x, i}(v t) \cdot \exp \left[-j \frac{4 \pi}{\lambda} r_{0}\right] \\
& \cdot \exp \left[-j \frac{\pi \Delta x_{i}^{2}}{2 \lambda r_{0}}\right] \cdot \exp \left[-j \frac{2 \pi v^{2}}{\lambda} \frac{\left(t-\frac{\Delta x_{i}}{2 v}\right)^{2}}{r_{0}}\right] .
\end{aligned}
$$

Here, the first exponential describes a constant phase offset for a given slant range $r_{0}$ that is equal for all receivers, while the second exponential accounts for an additional constant phase offset that is due to the different along-track displacements between each individual receiver and the transmitter. The timevarying azimuth modulation of the bistatic SAR is then given by the third exponential. By comparing (6) with the point target response of a monostatic SAR

$$
\begin{aligned}
h_{m}(t) \cong \tilde{A}_{T x}(v t) \cdot & \tilde{A}_{R x}(v t) \\
\cdot & \cdot \exp \left[-j \frac{4 \pi}{\lambda} r_{0}\right] \cdot \exp \left[-j \frac{2 \pi v^{2} t^{2}}{\lambda r_{0}}\right]
\end{aligned}
$$

it becomes clear that the bistatic azimuth response evolves from its monostatic counterpart by a time delay $\Delta t=\Delta x_{i} / 2 v$ and a phase shift $\Delta \varphi=-\pi \Delta x_{i}^{2} / 2 \lambda r_{0}$ if we substitute the antenna footprints $A_{T x}$ and $A_{R x}$ by $\tilde{A}_{T x}(v t)=A_{T x}\left(v t+\Delta x_{i} / 2\right)$ and $\tilde{A}_{R x}(v t)=A_{R x}\left(v t+\Delta x_{i} / 2\right)$. The bistatic antenna footprint is then given by

$$
\begin{aligned}
A_{i}(v t) & =\tilde{A}_{T x}(v t) \cdot \tilde{A}_{R x}(v t) \\
& =A_{T x}\left(v t+\frac{\Delta x_{i}}{2}\right) \cdot A_{R x}\left(v t+\frac{\Delta x_{i}}{2}\right) .
\end{aligned}
$$




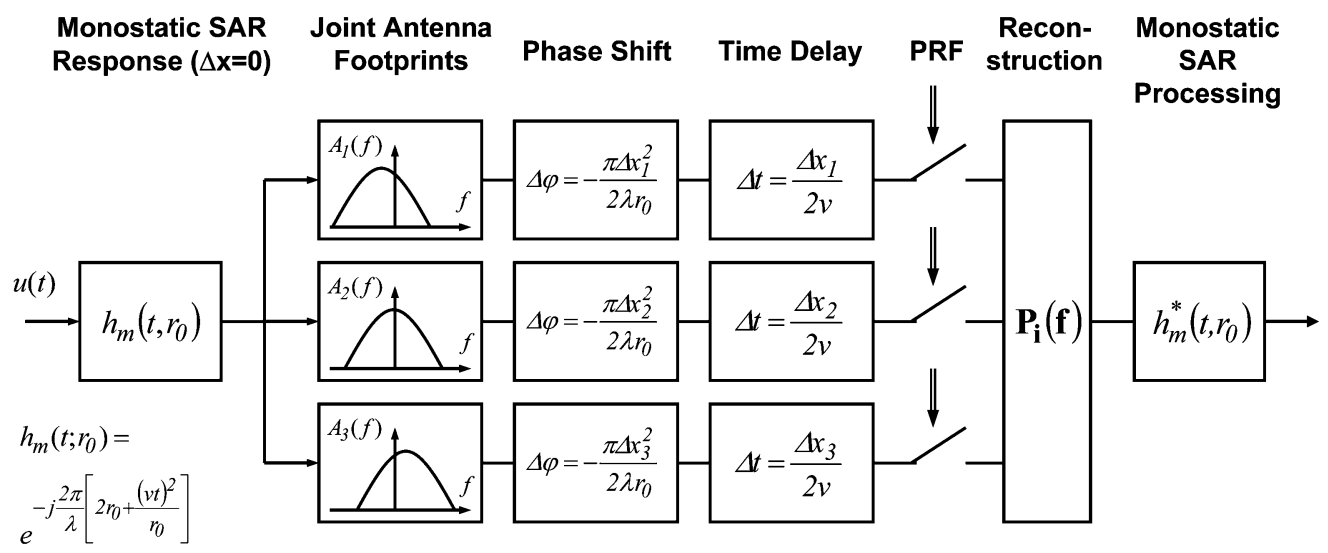

Fig. 3. Quadratic phase approximation.

Therefore, the multiple aperture system can be regarded as a monostatic SAR that is followed by additional time and phase shifts for each receiver channel. This is illustrated in Fig. 3. Since the joint antenna footprint may be different for each receiver, we have introduced a separate amplitude weighting of the azimuth spectrum in each channel, but it is also possible to separate the joint antenna pattern in two parts, where a first weighting function in the monostatic SAR response accounts for a weighting of the Doppler spectrum, which is common to all receivers, and the second transfer function in each channel accounts for residual deviations between the individual receivers. These deviations may for example result from different Doppler centroids due to the different along-track displacements, inaccuracies in the antenna pointing, or a mutual coupling between the antenna subarrays in case of a single-platform DPC system. It may be expected that such a separation in two transfer functions will have an impact on the stability of the signal reconstruction, but this topic deserves further investigation.

\section{ANALYTIC EXAMPLE}

As a simple example for an analytic derivation of the reconstruction filters we consider a bistatic SAR with two receiver channels having the same azimuth antenna pattern. This two-channel system may serve as an example for the unambiguous recovery of the Doppler spectrum resulting from a SAR signal recorded by the split antenna technique in TerraSAR-X and Radarsat 2. Since it is possible to incorporate the transfer function due to the antenna pattern into the monostatic SAR response, the remaining difference between the two channels is given by the two impulse responses

$$
h_{1}\left(t ; \Delta x_{1}\right)=\exp \left[-j \frac{\pi \Delta x_{1}^{2}}{2 \lambda r_{0}}\right] \cdot \delta\left(t-\frac{\Delta x_{1}}{2 v}\right)
$$

TABLE I

SimUlation PARAMETERS FOR DUAL RECEIVE ANTENNA

\begin{tabular}{lc}
\hline \hline Wavelength & $3.1 \mathrm{~cm}$ \\
PRF & $3600 \mathrm{~Hz}$ \\
Velocity & $7600 \mathrm{~m} / \mathrm{s}$ \\
Antenna Length (Tx) & $2.4 \mathrm{~m}(4.8 \mathrm{~m} / 2)$ \\
Number of Subapertures (Rx) & 2 \\
Sub-Aperture Length (Rx) & $2.4 \mathrm{~m}(4.8 \mathrm{~m} / 2)$ \\
Slant Range & $700 \mathrm{~km}$ \\
Inter-Channel SNR & $20 \mathrm{~dB}$ \\
\hline \hline & \\
$h_{2}\left(t ; \Delta x_{2}\right)=\exp \left[-j \frac{\pi \Delta x_{2}^{2}}{2 \lambda r_{0}}\right] \cdot \delta\left(t-\frac{\Delta x_{2}}{2 v}\right)$.
\end{tabular}

In the frequency domain, these impulse responses correspond to

$$
\begin{aligned}
& H_{1}\left(f ; \Delta x_{1}\right)=\exp \left[-j \frac{\pi \Delta x_{1}^{2}}{2 \lambda r_{0}}\right] \cdot \exp \left(-j 2 \pi \frac{\Delta x_{1}}{2 v} f\right) \\
& H_{2}\left(f ; \Delta x_{2}\right)=\exp \left[-j \frac{\pi \Delta x_{2}^{2}}{2 \lambda r_{0}}\right] \cdot \exp \left(-j 2 \pi \frac{\Delta x_{2}}{2 v} f\right) .
\end{aligned}
$$

The matrix $\mathbf{H}(\mathbf{f})$ is then given by (13), shown at the bottom of the page, and the inverse matrix $\mathbf{H}^{-\mathbf{1}}(\mathbf{f})$ can be derived as in (14), shown at the bottom of the page. The transfer functions of the reconstruction filters $P_{1}(f)$ and $P_{2}(f)$ are then

$$
P_{1}(f)= \begin{cases}\frac{\exp \left(\left(j \pi \Delta x_{1}^{2}\right) / 2 \lambda r_{0}\right) \cdot \exp \left(\left(j \pi \Delta x_{1} f\right) / v\right)}{1-\exp \left[\left(j \pi \cdot \mathrm{PRF} \cdot\left(\Delta x_{2}-\Delta x_{1}\right)\right) / v\right]}, & -\mathrm{PRF}<f<0 \\ \frac{\exp \left(\left(j \pi \Delta x_{1}^{2}\right) / 2 \lambda r_{0}\right) \cdot \exp \left(\left(j \pi \Delta x_{1} f\right) / v\right)}{1-\exp \left[\left(j \pi \cdot \mathrm{PRF} \cdot\left(\Delta x_{1}-\Delta x_{2}\right)\right) / v\right]}, & 0<f<\mathrm{PRF}\end{cases}
$$

$$
\mathbf{H}(\mathbf{f})=\left[\begin{array}{cr}
\exp \left[-j \pi\left(\frac{\Delta x_{1}^{2}}{2 \lambda r_{0}}+\frac{\Delta x_{1}}{v} f\right)\right] & \exp \left[-j \pi\left(\frac{\Delta x_{2}^{2}}{2 \lambda r_{0}}+\frac{\Delta x_{2}}{v} f\right)\right] \\
\exp \left[-j \pi\left(\frac{\Delta x_{1}^{2}}{2 \lambda r_{0}}+\frac{\Delta x_{1}}{v}(f+\mathrm{PRF})\right)\right] & \exp \left[-j \pi\left(\frac{\Delta x_{2}^{2}}{2 \lambda r_{0}}+\frac{\Delta x_{2}}{v}(f+\mathrm{PRF})\right)\right]
\end{array}\right]
$$

$$
\mathbf{H}^{-1}(\mathbf{f})=\frac{1}{\exp \left(-j \pi \frac{\Delta x_{2}}{v} \mathrm{PRF}\right)-\exp \left(-j \pi \frac{\Delta x_{1}}{v} \mathrm{PRF}\right)}\left[\begin{array}{cc}
\exp \left[j \pi\left(\frac{\Delta x_{1}^{2}}{2 \lambda r_{0}}+\frac{\Delta x_{1}}{v} f-\frac{\Delta x_{2}}{v} \mathrm{PRF}\right)\right] & -\exp \left[j \pi\left(\frac{\Delta x_{1}^{2}}{2 \lambda r_{0}}+\frac{\Delta x_{1}}{v} f\right)\right] \\
-\exp \left[j \pi\left(\frac{\Delta x_{2}^{2}}{2 \lambda r_{0}}+\frac{\Delta x_{2}}{v} f-\frac{\Delta x_{1}}{v} \mathrm{PRF}\right)\right] & \exp \left[j \pi\left(\frac{\Delta x_{2}^{2}}{2 \lambda r_{0}}+\frac{\Delta x_{2}}{v} f\right)\right]
\end{array}\right]
$$



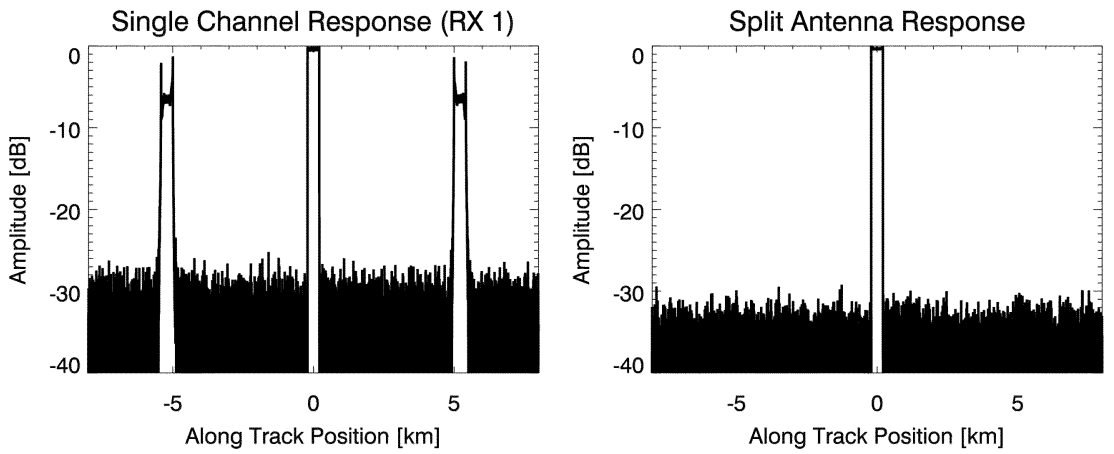

Fig. 4. (Left) Azimuth response for one subaperture and (right) after nonuniform DPCA reconstruction.
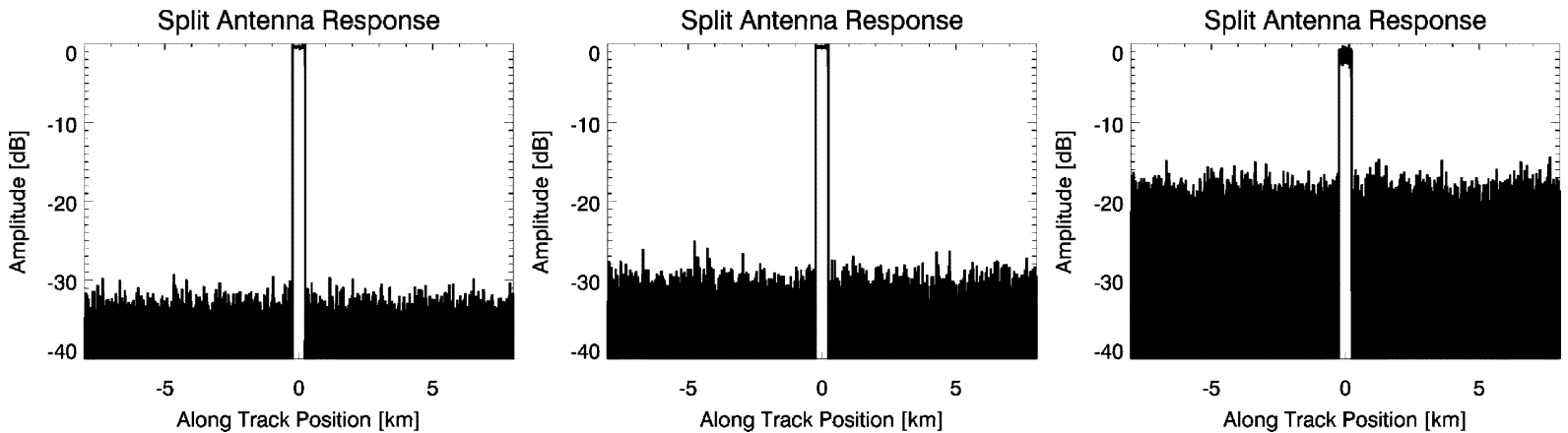

Fig. 5. Reconstruction for different intersample offsets. (Left) $100 \%$ (uniform sampling with $2.4 \mathrm{~m} / 2.4 \mathrm{~m}$ ). (Middle) $33 \%(1.2 \mathrm{~m} / 3.6 \mathrm{~m})$. (Right) $5 \%$ (0.24m/4.56m).

$P_{2}(f)= \begin{cases}\frac{\exp \left(\left(j \pi \Delta x_{2}^{2}\right) / 2 \lambda r_{0}\right) \cdot \exp \left(\left(j \pi \Delta x_{2} f\right) / v\right)}{1-\exp \left[\left(j \pi \cdot \mathrm{PRF} \cdot\left(\Delta x_{1}-\Delta x_{2}\right)\right) / v\right]}, & -\mathrm{PRF}<f<0 \\ \frac{\exp \left(\left(j \pi \Delta x_{2}^{2}\right) / 2 \lambda r_{0}\right) \cdot \exp \left(\left(j \pi \Delta x_{2} f\right) / v\right)}{1-\exp \left[\left(j \pi \cdot \mathrm{PRF} \cdot\left(\Delta x_{2}-\Delta x_{1}\right)\right) / v\right]}, & 0<f<\mathrm{PRF} .\end{cases}$

The numerator of the reconstruction filters can be regarded as compensating the different time delays and phase shifts within each branch, while the conjugate mirror structure in the denominator is responsible for a cancelation of the ambiguous frequencies within each branch. It becomes also clear that the denominator will vanish for $\Delta x_{2}=\Delta x_{1}$ and a reconstruction becomes impossible due to the coinciding samples in azimuth.

\section{Simulation Results}

In this section, some simulation results for the nonuniform DPCA reconstruction will be given. As an example, the dual receive antenna in TerraSAR-X [6] has been chosen. The relevant system parameters are summarized in Table I.

Fig. 4 shows the results for a reconstruction from nonuniform DPCA sampling. For better illustration, an extended scatterer has been chosen in this simulation. Furthermore, independent white noise has been added to each subaperture signal to account for differences in the two receiver channels. It becomes clear that the signal from each channel is strongly ambiguous (left), while the coherent reconstruction of the two subsampled SAR signals will provide a efficient ambiguity suppression (right). Further investigations were carried out to evaluate the sensitivity of the reconstruction result on the relative sample offset. Fig. 5 shows reconstruction examples for different degrees of nonuniformity in the azimuth sampling process. The intersample offset is given in percent, where $100 \%$ corresponds to the optimum offset with uniform sampling and $0 \%$ corresponds to coinciding samples. The results in Fig. 5 indicate an increasing noise floor in the reconstructed image for decreasing sampling offsets. Note that there are no ambiguities even for a strong deviation from the uniform sampling distance and the noise floor will only become significant in case of a rather strong nonuniform sampling. Further simulation results of the nonuniform DPC reconstruction have been presented in [11], where a multistatic satellite array consisting of three receivers has been assumed (cf. Fig. 1, bottom). This simulation shows that an unambiguous reconstruction of the SAR signal is also possible in case of large intersatellite separations and different Doppler centroids for each receiver.

The reconstruction algorithm has also been tested with real SAR data acquired by the DLR E-SAR system. Since there is currently no displaced phase center mode in the E-SAR system, monostatic data were used in this example. The data, which have an original sampling frequency $f_{s}$, were filtered with an ideal lowpass of bandwidth $B=f_{s} / 10$ to get SAR data $u(t)$ oversampled by a factor of 10 . Then, the ambiguous inputs to the two DPCA channels have been formed by taking every 20th sample for each channel. Selecting adjacent samples for the two channels will result in a maximum nonuniform sampling, while a distance of ten samples will yield a uniform sampling. After filtering with $P_{j}$ and coherent summation, the monostatic SAR processing follows. Fig. 6 compares the reconstruction result for a maximum nonuniform sampling with the SAR image of one ambiguous channel. It is clear, that the ambiguities are well suppressed in the image after nonuniform DPCA reconstruction.

\section{DISCUSSION}

An innovative algorithm for the suppression of azimuth ambiguities in case of nonuniform DPCA sampling has been derived. This reconstruction algorithm can be regarded as a time-variant digital beamforming on receive, which combines the individual receiver signals in a linear space-time processing. The algorithm is directly applicable to systems relying on the displaced 

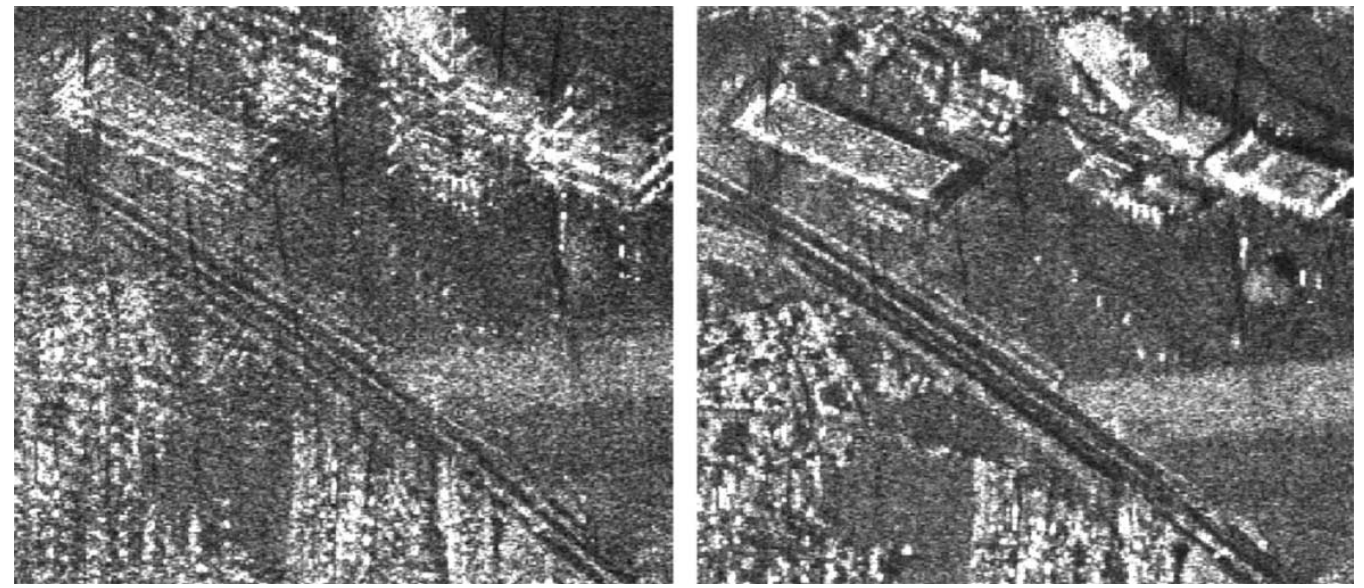

Fig. 6. (Left) Ambiguous image for one channel and (right) reconstruction after nonuniform DPC sampling.

phase center technique, like the Quad Array [2] and HRWS [3] SAR or the dual receive antenna approaches in TerraSAR-X [6] and Radarsat 2 [5], thereby avoiding any stringent PRF restriction. The algorithm will also be useful for ambiguity suppression in a distributed SAR with multiple receiver satellites. However, any cross-track separation of the receivers will introduce an additional phase in the received signals, which has to be compensated, e.g., via the simultaneous acquisition of a digital elevation model in case of multiple satellites [12], [13]. The joint processing of these data will then lead to a nonlinear combination of cross-track interferometry and digital beamforming on receive [7]. This combination will enable a distributed aperture SAR with small antennas to serve a broad spectrum of different applications [14], [15]. It is clear that such a multifunctional SAR system will require further and more detailed investigations. This short letter has to be regarded as a first step in this direction.

\section{ACKNOWLEDGMENT}

The authors thank the anonymous reviewers for their valuable comments and suggestions to improve the paper.

\section{REFERENCES}

[1] A. Currie and M. A. Brown, "Wide-swath SAR," Proc. Inst. Elect. Eng.-Radar, Sonar, Navigat., vol. 139, no. 2, pp. 122-135, 1992.
[2] G. D. Callaghan and I. D. Longstaff, "Wide swath spaceborne SAR using a quad element array," Proc. Inst. Elect. Eng.-Radar, Sonar, Navigat., vol. 146, no. 3, pp. 159-165, 1999.

[3] M. Suess, B. Grafmüller, and R. Zahn, "A novel high resolution, wide swath SAR system," in Proc. IGARSS, 2001.

[4] M. Younis, C. Fischer, and W. Wiesbeck, "Digital beamforming in SAR systems," IEEE Trans. Geosci. Remote Sensing, vol. 41, pp. 1735-1739, July 2003.

[5] L. Brule and H. Baeggli, "Radarsat-2 program update," in Proc. IGARSS, 2002.

[6] J. Mittermayer and H. Runge, "Conceptual studies for exploiting the TerraSAR-X dual receiving antenna," in Proc. IGARSS, 2003.

[7] G. Krieger and A. Moreira, "Potentials of digital beamforming in bi- and multistatic SAR," in Proc. IGARSS, 2003.

[8] N. A. Goodman, S. C. Lin, D. Rajakrishna, and J. M. Stiles, "Processing of multiple-receiver spaceborne arrays for wide area SAR," IEEE Trans. Geosci. Remote Sensing, vol. 40, pp. 841-852, Apr. 2002.

[9] J. P. Aguttes, "The SAR train concept: Required antenna area distributed over $N$ smaller satellites, increase of performance by $N$," in Proc. IGARSS, 2003.

[10] J. L. Brown, "Multi-channel sampling of low-pass signals," IEEE Trans. Circuits Syst., vol. 28, no. 2, pp. 101-106, 1981.

[11] G. Krieger, H. Fiedler, M. Rodriguez, D. Hounam, and A. Moreira, "System concepts for Bi- and multistatic SAR missions," in Proc. Int. Radar Symp., Dresden, Germany, June 2003, pp. 331-339.

[12] D. Massonnet, "Capabilities and limitations of the interferometric cartwheel," IEEE Trans. Geosci. Remote Sensing, vol. 39, pp. 506-520, Mar. 2001.

[13] G. Krieger, H. Fiedler, J. Mittermayer, K. Papathanassiou, and A. Moreira, "Analysis of multistatic configurations for spaceborne SAR interferometry," Proc. Inst. Elect. Eng.-Radar, Sonar, Navigat., vol. 150, no. 3, pp. 87-96, 2003.

[14] M. Martin, P. Klupar, S. Kilberg, and J. Winter, "Techsat 21 and revolutionizing space missions using microsatellites," in Proc. 15th Amer. Institute Aeronautics Astronautics Conf. Small Satellites, 2001.

[15] G. Krieger, H. Fiedler, and A. Moreira, "Bi- and multistatic SAR: Potentials and challenges," in Proc. EUSAR, 2004. 